\title{
Complete gastric outlet obstruction following acid ingestion complicated by acute pancreatitis and disseminated intravascular coagulation
}

\author{
Zahoor Ahmed, Zahoor Mohyuddin
}

\begin{abstract}
Summary
A case is described where accidental acid ingestion resulted in the development of oesophageal stricture and complete gastric outlet obstruction. Following a smoothly conducted pre-surgery endoscopic examination the patient developed acute pancreatitis, which, on initial clinical examination, was diagnosed as a viscus perforation. The severity of pancreatitis was such as to lead to disseminated intravascular coagulopathy and ultimately death. The interest lies in the fact that pancreatitis was precipitated in a previously traumatised stomach by such an innocuous procedure as fibre-optic endoscopy.
\end{abstract}

Keywords: acid ingestion, endoscopy, pancreatitis, gastric outlet obstruction, disseminated intravascular coagulation

The occurrence of gastric outlet obstruction is not a rare event as such, it most commonly occurs secondary to peptic ulcer disease or malignancy. Ingestion of corrosive chemical solutions is well known to cause injury to the oesophagus, stomach and duodenum, and, depending upon the extent and degree of burns, may lead to fibrotic changes culminating in complete gastric outlet obstruction. A case is reported in which acid ingestion resulted in such an outcome and progressed to the development of the uncommon but fatal complications of acute pancreatitis and disseminated intravascular coagulation, following endoscopy.

\section{Case report}

A 48-year-old man was admitted to hospital

Department of Thoracic and Vascular Surgery, Mafraq Hospital, Abu Dhabi, UAE Z Ahmed

Z Mohyuddin

Correspondence to Zahoor Ahmed, FRCS, FICS, FACA, PO Box 28233, Abu Dhabi, United Arab Emirates

Accepted 21 August 1996 the stomach into two compartments. Because of the worsening of symptoms, a repeat barium meal examination was done six weeks (figure 3) after the initial injury and this showed a complete mid-gastric obstruction. The oesophageal stricture was dilated endoscopically with a $15 \mathrm{~mm}$ balloon and the patient was started on total parenteral nutrition.

Two weeks later the endoscopy was repeated to re-assess the status of the oesophageal stricture prior to conducting a permanent drainage procedure for gastric obstruction. The oesophageal stricture was found to be quite pliable and the endoscope was admitted with ease. The day following this endoscopy the patient's condition suddenly deteriorated. He developed chest pain, tachycardia, tachypnoea and signs of dehydration. Further investigation revealed metabolic acidosis, marked leukocytosis, a low platelet count and a low serum calcium. His amylase at this stage was $205 \mathrm{U} / 1$. His cardiac evaluation was normal and so were the arterial blood gases. Later on the same day he developed abdominal fullness, with epigastric tenderness, guarding and absent bowel sounds. By this time he had also developed pyrexia. An abdominal X-ray showed a ground

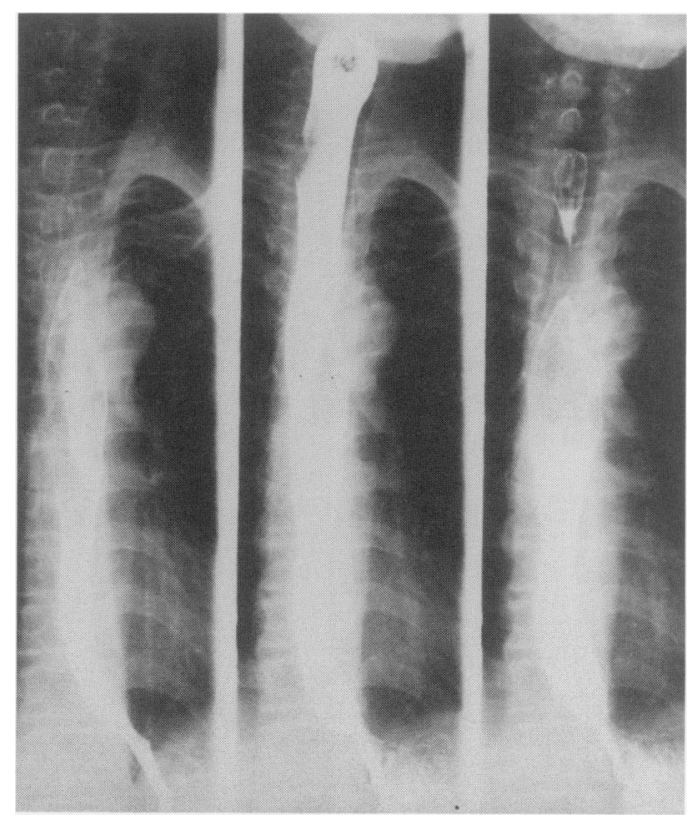

Figure 1 Barium swallow (done three weeks from the day of ingestion of acid) showing distal oesophageal stricture mid-gastric region (figure 2). Endoscopy revealed marked ulceration and destruction of gastroduodenal mucosa and a band dividing 
glass appearance indicating presence of free fluid. Abdominal ultrasound confirmed the presence of free fluid in the peritoneal cavity; the liver and spleen were normal but the pancreas could not be visualised. These findings were suggestive of a viscus perforation, possibly precipitated by the endoscopy. However, a gastrograffin study showed no leak from the oesophagus or stomach. An exploratory laparotomy was performed. The peritoneal cavity contained sero-sanguinous fluid and there were some adhesions in the supracolic compartment. The distal half of the stomach was found to be completely fibrosed. The pancreas was severely oedematous and there were areas of fat necrosis. There was no perforation of the stomach. Surgery had to be terminated before a gastric drainage procedure could be established because of hypotension

Figure 2 Barium meal (done three weeks from the day of ingestion of acid) showing mid-gastric stricture but free passage of contrast into the duodenum

Figure 3 Barium meal (done six weeks from the day of ingestion of acid) showing distal oesophageal stricture and complete gastric outlet obstruction

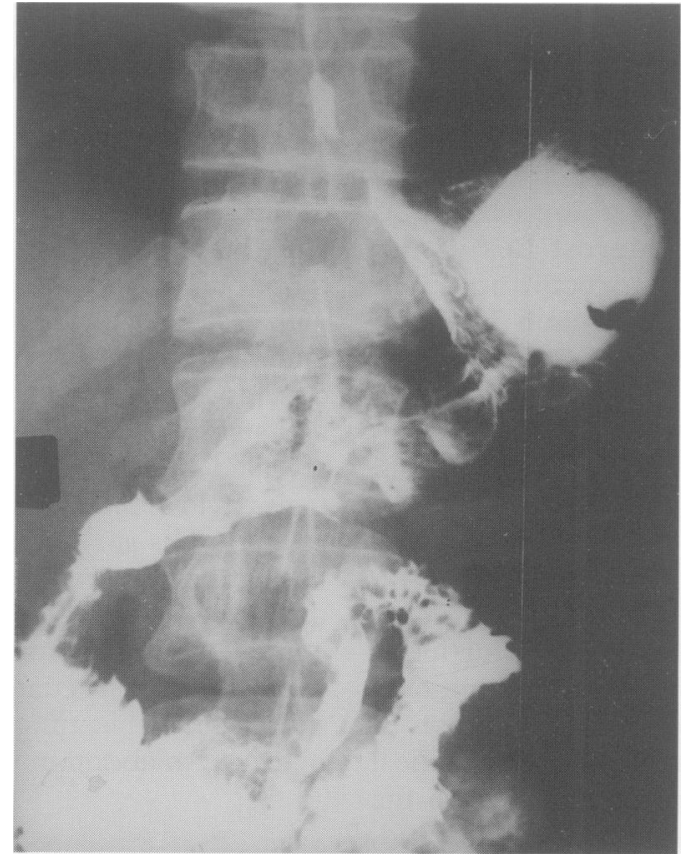

and bleeding due to disseminated intravascular coagulopathy.

Following surgery, his condition continued to deteriorate with upper gastrointestinal bleeding, jaundice, thrombocytopenia, elevated fibrinogen degradation products, elevated transaminases and increased partial thromboplastin time. Despite intensive management, including platelets and fresh frozen plasma transfusions, he progressed to multiorgan failure and died three days after laparotomy.

\section{Discussion}

Chemical injury to the oesophagus and stomach occurs as a result of accidental poisoning or attempted suicide with strong acids or alkalis. Alkaline solutions such as lye are more commonly swallowed, as they are generally tasteless and odourless and do not provoke protective reflexes. Acid solutions have an unpleasant taste and cause pain, and are difficult to swallow accidentally.

The injury caused by chemicals has been classified according to the degree of injury (box 1). ${ }^{1}$ It has been the belief that acids cause minor damage to the oesophagus owing to their rapid transit, and maximum damage to the stomach. However, in a recent study of 41 patients with acid injury, the stomach and oesophagus were equally involved, and $80.5 \%$ of patients had injury to both. ${ }^{2}$ In their series, Zargar et $a l^{2}$ recorded one fatal case of necrotising pancreatitis due to perforation of the stomach.

Several advances have occurred recently in the management of acid injury to the oesophagus. A carefully carried out endoscopy using a paediatric-sized flexible endoscope is safe, provides useful information regarding the grade and extent of injury, and helps to plan treatment. In a prospective study of 81 patients, Zargar et $a l^{1}$ performed the first endoscopy within 36 hours in all patients. Patients with radiological demonstration of perforation were not scoped. No perforation was caused by endoscopy. On the basis of endoscopy findings the authors stated that there were no late sequelae in grade 1 or 2 injuries. Patients with grade $2 b$ and 3 injuries

\begin{tabular}{|c|}
\hline $\begin{array}{l}\text { Modified endoscopic classification of } \\
\text { burns }^{1}\end{array}$ \\
\hline 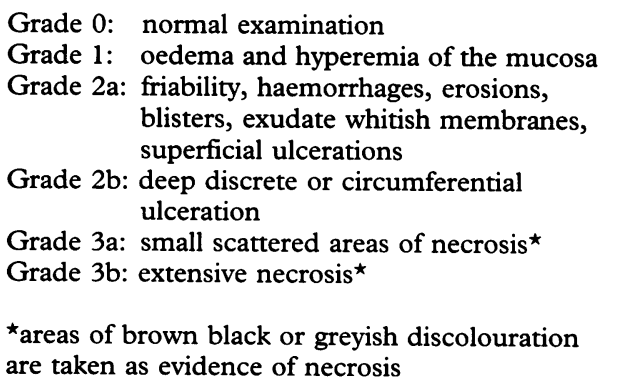 \\
\hline
\end{tabular}


developed strictures. Extensive grade 3 injuries carry a high mortality, and could only be salvaged by emergency oesophagectomy.

There is one recent randomised trial to assess the beneficial role of steroids in preventing cicatricial contractures. ${ }^{3}$ Steroids failed to prevent development of oesophageal strictures in children who had ingested a caustic substance. In another study in 202 children $^{4}$ it was found that most caustic oesophageal strictures could be treated by conservative measures, the chances of success being higher if the caustic agent was not lye, the age was less than eight years, stricture length less than $5 \mathrm{~cm}$ and involving the upper third.

The unusual feature of our patient is the terminal event of acute pancreatitis precipitated by endoscopy. It is possible that the initial injury had produced a gastric perforation and

\footnotetext{
1 Zargar SA, Kochhar R, Mehta S, Mehta SK. The role of fibreoptic endoscopy in the management of corrosive ingestion and modified endoscopic classification of burns. ingestion and modified endoscopic

2 Zargar SA, Kochhar R, Nagi B, Mehta S, Mehta SK.

Zargar SA, Kochhar R, Nagi B, Mehta S, Mehta SK.
Ingestion of corrosive acids. Spectrum of injury to upper gastrointestinal tract and natural history. Gastroenterology 1989; 97: 702-7.

3 Anderson KD, Rouse TM, Randolph JG. A controlled trial of corticosteroids in children with corrosive injury of esophagus. $N$ Engl $\mathcal{F}$ Med 1990; 323: 637-40.

4 Gundogdu HZ, Tanyel FC, Buyukpamukcu N, Hicsonmez A. Conservative treatment of caustic oesphageal strictures in children. $\mathcal{F}$ Pediatr Surg 1992; 27: 767-70.

5 Rifat L, McIntosh JK, Dudrick SJ. Nutritional management

of acute and chronic pancreatitis. Surg Clin North Am 1991; 71: $579-95$.
}

\section{Learning points}

- contrary to previous belief, acids are equally injurious to oesophagus and stomach

- delayed onset of pancreatitis following gastric corrosive injury is always a possibility

Box 2

pancreatitis, which made the pancreas susceptible to inflammation. It is also possible that hyperlipidaemia due to parenteral nutrition contributed to pancreatitis. ${ }^{5}$ The disparity between the clinical symptoms and severity of pancreatitis is also well known. ${ }^{6}$ The onset of disseminated intravascular coagulopathy in this patient is a known complication of severe pancreatitis. ${ }^{7,8}$

6 Teng RJ, Tsau YK, Chang MH, Lee CY, Chen CL. An autopsy case of Reye's syndrome associated with acute pancreatitis, acute renal failure and disseminated intravascular coagulopathy. Taiwan I Hseuh TSA Chih (f Formosan Med Assoc) 1990; 89: 887-91.

7 Murakawa M, Okamura T, Shibuya T, Harada M, Otsuka $T$, Niho Y. Use of synthetic protease inhibitor for the treatment of asparaginase induced acute pancreatitis complicated by disseminated intravascular coagulation. Ann Haematol 1992; 64: 249-52.

8 Ogawa $M$. Coagulation abnormalities and disseminated intravascular coagulation in severe acute pancreatitis. Nippon-Rinsho (Fpn $\mathcal{F}$ Clin Med) 1990; 48: 140-7.

\title{
Paraplegia due to thoracic disc herniation
}

\author{
Badal Pal, Andrew Johnson
}

\begin{abstract}
Summary
Disc herniation at the thoracic the spine level is more common than generally thought. Localisation of pain may be vague and may erroneously point to cardiopulmonary, gastrointestinal, genito-urinary or even psychiatric disease. Magnetic resonance imaging is the investigation of choice, especially if spinal cord compression is suspected.
\end{abstract}

Keywords: disc herniation, paraplegia, spinal cord compression, magnetic resonance imaging

Withington Hospital, Nell Lane, Didsbury, Manchester M20 2LR, UK

B Pal

A Johnson

Accepted 25 September 1996
Disc herniation at the thoracic spine level has been thought to be an uncommon problem until recently. Although in life less than $1 \%$ incidence has been quoted, autopsy series show a higher incidence of thoracic disc herniations of between 7 and $15 \% .^{1,2}$ Most series of patients with dorsal disc herniations suggested incidences under $2 \%$ of all operated discs. ${ }^{1,2,7}$ It has been postulated that a number of factors at the thoracic spine level are responsible for the low overall incidence of thoracic disc herniation, for example, the relatively limited mobility of the thoracic spine secondary to the small sizes of the thoracic discs, the relative restraint of the thoracic spine because of the ribs and sternum, and the orientation of the facetal joints in the coronal plane. ${ }^{2}$ The variable clinical manifestations can obscure the diagnosis, and until recently the lack of radiographic sensitivity and specificity for assessment of complaints referable to the thoracic spine had compounded the issue. ${ }^{2}$ We wish to report on a disc herniation at the thoracic spine level in a patient whose presenting features were vague or confusing enough to suggest an initial diagnosis of a functional problem by a number of physicians, including casualty doctors, a consultant neurologist, and a consultant radiologist, until paraplegia developed due to cord compression. 